\title{
A composição gravimétrica como ferramenta fundamental na gestão dos resíduos sólidos: estudo de caso do município de Rio Verde/GO
}

A sociedade contemporânea enfrenta um dilema entre a geração desenfreada de resíduos sólidos, sua disposição e os impactos ambientais que causam. Assim vê-se a necessidade de identificar o perfil dos resíduos gerados em um local, para que se possa planejar a melhor forma de gerenciá-los, atendendo às exigências da PNRS, sendo o estudo da composição gravimétrica uma ferramenta para se conhecer esse perfil. O presente estudo objetivou determinar e avaliar a porcentagem das frações dos materiais que compõem os resíduos sólidos urbanos de Rio Verde, afim de fornecer informações visando a melhoria da gestão dos resíduos. Foi adotado o procedimento metodológico que consiste na homogeneização quarteamento dos resíduos para obtenção da amostra significativa a ser pesada. Como principais resultados a matéria orgânica $(55,06 \%)$ foi a mais representativa, seguida pelos materiais passíveis de reciclagem, plásticos (17,94\%), papel/papelão $(12,88 \%)$, vidro $(2,49 \%)$ e metais $(1,94 \%)$, além dos rejeitos $(4,78 \%)$, percentuais similares às médias nacionais. Os resultados também demonstraram que determinadas rotas possuem maior propensão a resíduos específicos, sendo as áreas centrais as maiores geradoras de resíduos passíveis de reciclagem. Portanto, a partir da composição gravimétrica é possível obter informações que auxiliem no processo de tomada de decisão, considerando as particularidades das diferentes regiões que compõe o município.

Palavras-chave: Gravimetria; Resíduos sólidos urbanos; Caracterização física.

\section{Gravimetric composition as fundamental tool in solid waste management: case study in the municipality of Rio Verde/GO}

\begin{abstract}
Our contemporary society faces a dilemma between the ever-increasing generation of solid waste, its disposal and the environmental impact they cause. Therefore, it is necessary to identify the profile of the waste generated in one place in order to plan the best way to manage them, complying with the PNRS (National Policy for Solid Waste) requirements. One of the tools to identify this profile is the study of the gravimetric composition. The present study aimed at determining and evaluating the percentage of materials fractions which compose the urban solid waste of Rio Verde, to provide information that help waste management. The methodological procedure adopted consists in the homogenization and quartering of the waste to obtain the sample to be weighted. As main results, organic matter $(55.06 \%)$ was the most representative of the main results, followed by the materials selectable for recycling, plastic (17.94\%), paper/cardboard (12.88\%), glass $(2.49 \%)$ and metals $(1.94 \%)$, besides the residues $(4.78 \%)$. The results also showed that certain routes are prone to specific waste, with central are being the largest generators of recycled materials. Therefore, from the gravimetric composition, it is possible to obtain information to assist in the decision-making process, considering the particularities of the different regions that make up the municipality.
\end{abstract}

Keywords: Gravimetry; Urban Solid Waste; Physical Characterization

Topic: Engenharia da Sustentabilidade e Meio Ambiente

Reviewed anonymously in the process of blind peer
Received: 05/10/2020

Approved: $\mathbf{2 6 / 1 1 / 2 0 2 0}$
Bruno de Oliveira Costa Couto

Instituto Federal Goiano, Brasil

http://lattes.cnpq.br/7679222927811979

http://orcid.org/0000-0003-0319-3293

bruno.couto@ifgoiano.edu.br

Ananda Ferreira de Oliveira (iD)

Instituto Federal Goiano, Brasil

http://lattes.cnpq.br/4720132916541713

http://orcid.org/0000-0003-4851-2753

anandaferreira13@gmail.com

Bruno Botelho Saleh (iD

Instituto Federal Goiano, Brasil

http://lattes.cnpq.br/2279628061058840

http://orcid.org/0000-0002-6790-0690

bruno.saleh@ifgoiano.edu.br
Bertiê Bastos de Morais (iD

Instituto Federal Goiano, Brasil

http://lattes.cnpq.br/7584372167982700

http://orcid.org/0000-0003-1363-2915

bertiebastos@gmail.com

Clever Rosa da Silva Júnior

Instituto Federal Goiano, Brasil

http://lattes.cnpq.br/4442653271800123

clever.caldas@hotmail.com
Referencing this:

COUTO, B. O. C.; OLIVEIRA, A. F.; SALEH, B. B.; MORAIS, B. B.; SILVA JÚNIOR, C. R.. A composição gravimétrica como ferramenta fundamental na gestão dos resíduos sólidos: estudo de caso do município de Rio Verde/GO. Revista Ibero Americana de Ciências Ambientais, v.11, n.6, p.404-410, 2020. DOI:

http://doi.org/10.6008/CBPC2179-6858.2020.006.0033 


\section{INTRODUÇÃO}

A expansão e o aumento da densidade demográfica nas áreas urbanas culminam no surgimento de problemas gerados por resíduos sólidos, uma vez que a infraestrutura sanitária da maioria das cidades brasileiras não acompanha o ritmo acelerado de crescimento (REZENDE et al., 2013; SONTAG et al., 2015; PESSOA et al., 2018). A problemática da geração exponencial de resíduos tem preocupado a maior parte dos países, em função dos impactos ambientais negativos gerados por tal atividade. Um eficiente sistema municipal de gestão de resíduos sólidos urbanos deve prever a quantidade e a qualidade dos resíduos gerados pela sua população, para que essas informações forneçam suporte para o planejamento urbano, dimensionamento adequado de coletores, bem como na escolha das técnicas de tratamento e disposição final de resíduos sólidos mais adequadas.

Considerando a problemática da geração e disposição inadequada dos resíduos sólidos no Brasil foi elaborada a Política Nacional de Resíduos Sólidos (PNRS), que dentre outras diretrizes torna obrigatório a formulação do Plano Municipal de Gestão Integrada de Resíduos Sólidos (PMGIRS), sendo que este deverá ter em seu conteúdo o diagnóstico da situação quanto à geração de resíduos no local, indicando a origem, o volume, a caracterização e as formas de destinação e disposição final (BRASIL, 2010; DOMINGOS et al., 2015).

Neste contexto, as matérias recicláveis desempenham papel estratégico, pois: promovem a educação ambiental, redução do desperdício, diminuição dos custos relacionados ao transporte e disposição final, além do potencial para a geração de trabalho e renda nas cooperativas de reciclagem (FRÉSCA et al., 2008; MACHADO et al., 2019). Assim, existe a necessidade de implementação e incentivo as atividades de reciclagem, de maneira a valorar os resíduos descartados e, consequentemente, reduzindo os volumes enviados aos sistemas de disposição final (PASCHOALIN FILHO et al., 2014; PESSOA et al., 2018).

Assim, vê-se a necessidade de identificar o perfil dos resíduos gerados no município de Rio Verde, no intuito de planejar a melhor forma de gerenciá-los, atendendo às exigências da PNRS. Desta forma, a composição gravimétrica torna-se ferramenta fundamental para se conhecer esse perfil, demonstrando as porcentagens das frações de materiais que compõe os resíduos sólidos urbanos. Portanto, o presente estudo visa determinar e avaliar a composição dos diferentes materiais que compõem os resíduos sólidos domiciliares do município de Rio Verde, apontando também as principais medidas de gerenciamento e minimização dos impactos ambientais existentes.

\section{METODOLOGIA}

A área de estudo está localizada no município de Rio Verde (latitude: 17은 53" S e longitude: 50ำ $55^{\prime} 41$ " W), região sudoeste do estado de Goiás. A pesquisa em questão foi realizada no ano de 2018 , quando o município possui população estimada em 229.651 habitantes, segundo o IBGE Cidades.

A composição gravimétrica foi realizada com os resíduos sólidos urbanos oriundos da coleta convencional (domiciliares e comerciais) da área urbana, obtendo um total de 17 pesagens, cada uma corresponde a uma rota de coleta, com início no dia 06/03/2018 e fim no dia 22/03/2018. 
A partir da literatura existente, foi verificado que não existe consenso acerca do melhor método para segregação e caracterização dos resíduos. Assim, para este estudo, foi adotado procedimento metodológico adaptado de Frésca (2007), conforme esquematizado na Figura 1. Em razão do volume de resíduos, foi adotado procedimento de amostragem segundo a ABNT NBR 10.007/2004, que dispõe sobre a amostragem de resíduos sólidos.

O caminhão deposita os resíduos, formando a primeira pilha, sendo posteriormente homogeneizada coma a ajuda de uma pá carregadeira. Após esse processo são retirados de pontos aleatórios (do topo, meio e base da pilha) os resíduos, ainda nas sacolas plásticas, para formar a amostra composta. Estas parcelas são novamente homogeneizadas, de forma a se obter uma amostra homogênea - ABNT/NBR 10007 (ABNT, 2004).

Assim, a amostra composta consistiu em um monte de aproximadamente $200 \mathrm{~kg}$, que é espalhado sobre a lona plástica, onde, manualmente, os sacos plásticos são rasgados e os resíduos misturados para homogeneização (Figura 2). Após esse processo os resíduos foram separados em quatro montes de aproximadamente $50 \mathrm{~kg}$ cada, onde, para cada monte, foi feita a mistura manual e, em seguida, o processo de quarteamento, separando-os em quatro montes e desprezando metade, restando oito montes de aproximadamente $12,5 \mathrm{~kg}$ (Figura 2).

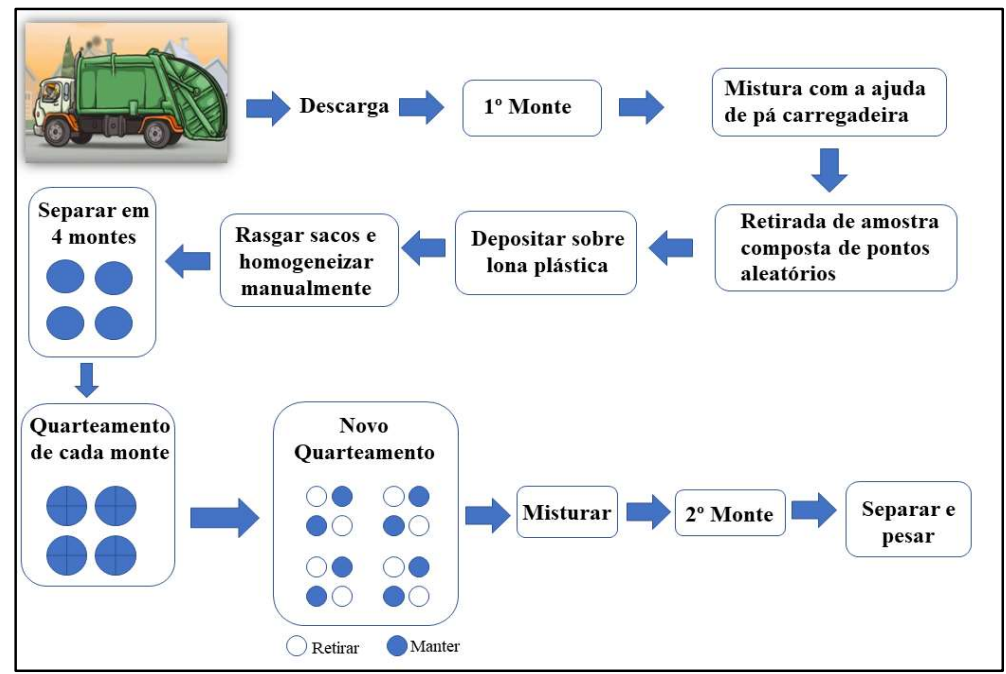

Figura 1: Metodologia para caracterização física dos resíduos sólidos.

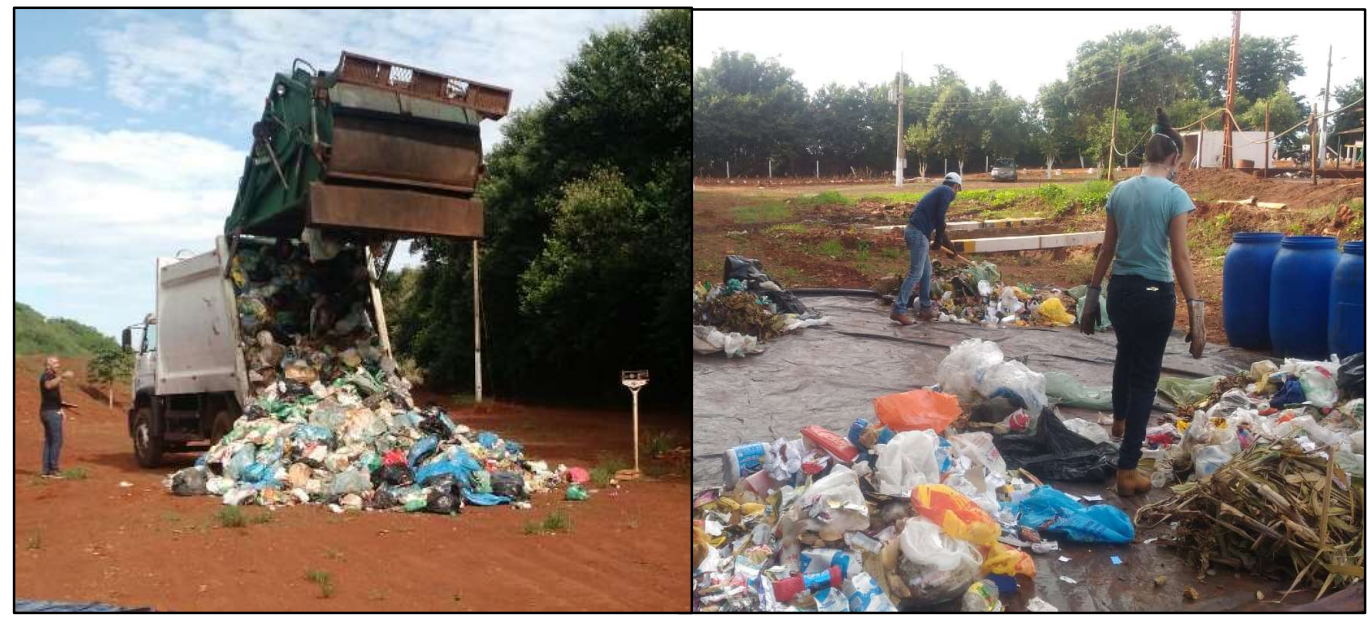

Figura 2: Composição da amostra segundo a metodologia adotada. 
Ao fim dessa etapa os resíduos foram misturados manualmente, formando um monte de $100 \mathrm{~kg}$, sendo esta quantidade suficiente para uma amostra representativa. O intervalo de 91 a $140 \mathrm{~kg}$ para o peso das amostras de resíduos sólidos é recomendado pela American Society for Testing and Materials (ASTM, 2008), sendo que valores fora dessa faixa apresentam discrepância significativa na amostra. Pode-se observar que, para este estudo, adotou-se $100 \mathrm{Kg}$.

O total de $100 \mathrm{~kg}$ de resíduos sólidos domiciliares restantes foram segregados em 7 bombonas de 200 litros, cada uma identificada com os grupos desejados, nesse caso: Matéria orgânica, papel e papelão, plásticos, metais, vidros, couro/trapo/pano/borracha e rejeitos.

\section{RESULTADOS E DISCUSSÃO}

Segundo dados fornecidos pela empresa responsável pela coleta, transporte e disposição final dos resíduos sólidos domiciliares de Rio Verde, a cada semana o aterro de Rio Verde recebe cerca de 1.000 toneladas de resíduos sólidos domiciliares. Na Figura 3 encontram-se os valores médios de geração de resíduos sólidos domiciliares de Rio Verde, obtidos a partir dos dados de pesagem diária.

A média diária de resíduos domiciliares coletados é de cerca de 166 toneladas (166.324,87 kg). Considerando-se que a população total estimada para o município em 2018 foi de 229.651 habitantes (IBGE, 2018) e que toda a população urbana do município (100\%) é atendida pela coleta, estima-se uma geração de 0,724 $\mathrm{kg} \mathrm{hab.}^{-1} \mathrm{dia}^{-1}$ de resíduos sólidos domiciliares. Esse valor, no entanto, tende a se modificar devido ao caráter dinâmico que tem demonstrado a evolução socioeconômica e populacional do município. Adicionalmente, ocorre maior produção de resíduos no início da semana (segunda e terça principalmente), devido ao acumulo de resíduos no final de semana e a ausência de coleta aos domingos.

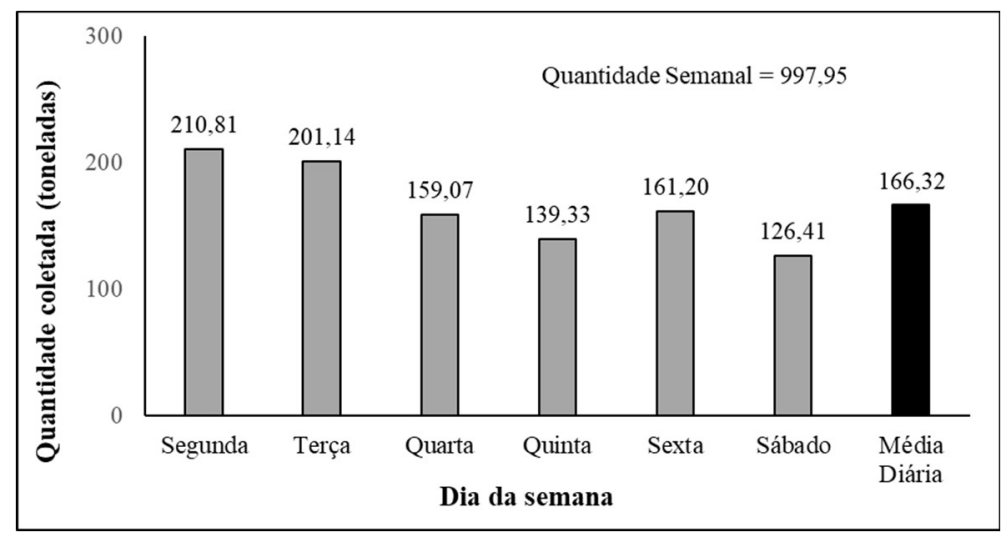

Figura 3: Quantificação da geração de RSD no município de Rio Verde.

O resultado da composição gravimétrica média dos resíduos sólidos urbanos do município de Rio Verde apresentados na Figura 4. Do total de resíduos sólidos encaminhados ao aterro sanitário de Rio Verde, a matéria orgânica $(55,12 \%)$ foi a mais representativa (sendo composta principalmente por restos de comida, galhos, folhas de árvore e grama), seguida pelos materiais passíveis de reciclagem, plásticos (17,93\%), papel/papelão $(12,67 \%)$, vidro $(2,50 \%)$ e metais $(1,94 \%)$. Também houve geração de rejeitos $(4,82 \%)$, principalmente fraudas descartáveis e resíduos de isopor. Os rejeitos são aqueles materiais que ainda não possuem tecnologia e/ou viabilidade para serem reciclados ou reaproveitados de alguma maneira. É 
importante salientar que as embalagens longa vida, também chamadas de embalagens cartonadas, foram consideradas no grupo papel/papelão, em função da sua geração ter sido menor do que a esperada e por possuir em sua composição $75 \%$ de papel e apenas $25 \%$ de outros materiais, como plástico e alumínio. Observando os valores obtidos nota-se que cerca de 90,31\% dos resíduos gerados em Rio Verde são passíveis de reaproveitamento, compostagem ou reciclagem.

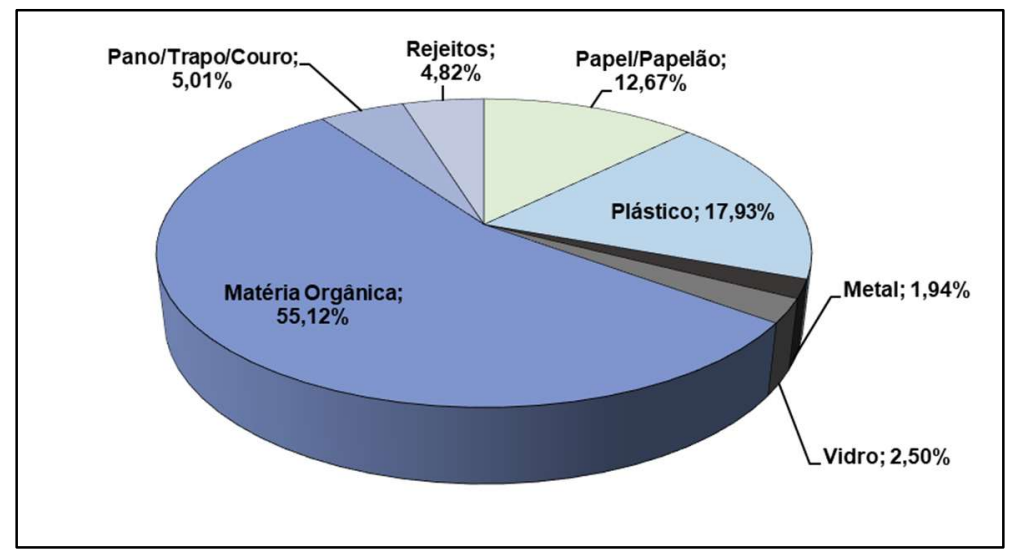

Figura 4: Composição gravimétrica dos RSD coletados em Rio Verde.

Os percentuais encontrados dos resíduos encontrados no município de Rio Verde são semelhantes aos do Plano Nacional de Resíduos Sólidos, o qual afirma percentual de matéria orgânica igual a 51,4 \% e material reciclável igual a 31,9 \% (considerando papel/papelão, plástico, metal e vidro). Vale ressaltar a quantidade elevada de panos, trapos e couro, provenientes de vestimentas encontradas em meio aos resíduos domiciliares, condição que levou ao surgimento de uma classe específica para este tipo de resíduos, sendo o mesmo equivalente a 5,01\% dos resíduos do município. Considerando algumas rotas em particular, estes resíduos chegaram a representar mais de $10 \%$ do total.

Entretanto, é interessante a analisar a composição dos resíduos para cada rota de coleta, pois devido as condições econômicas da população e ao zoneamento econômico de cada região, os hábitos de consumo e, consequentemente, os resíduos gerados são diferentes. Desta forma, na Tabela 1 são apresentados os resultados da composição gravimétrica dos resíduos, em sua respectiva rota. Vale ressaltar que este tipo de informação auxilia na gestão e tomada de decisões considerando as particularidades de cada região do município.

Tabela 1: Composição gravimétrica dos resíduos sólidos encaminhados ao aterro sanitário municipal de Rio Verde/GO no ano de 2018.

\begin{tabular}{lllllll}
\hline Rotas & $\begin{array}{l}\text { Papel/ } \\
\text { Papelão }\end{array}$ & Plástico & Metal & Vidro & Matéria Orgânica & $\begin{array}{l}\text { Pano/Trapo/ } \\
\text { Couro/Borracha }\end{array}$ \\
\hline $\mathbf{1}$ & $16,36 \%$ & $20,70 \%$ & $2,27 \%$ & $3,20 \%$ & $49,59 \%$ & $1,56 \%$ \\
\hline $\mathbf{2}$ & $6,72 \%$ & $14,45 \%$ & $0,74 \%$ & $0,46 \%$ & $61,72 \%$ & $11,50 \%$ \\
\hline $\mathbf{3}$ & $17,89 \%$ & $15,24 \%$ & $1,47 \%$ & $0,05 \%$ & $59,31 \%$ & $4,30 \%$ \\
\hline $\mathbf{4}$ & $8,83 \%$ & $15,50 \%$ & $0,71 \%$ & $2,54 \%$ & $67,62 \%$ & $0,87 \%$ \\
\hline $\mathbf{6}$ & $10,44 \%$ & $17,47 \%$ & $2,15 \%$ & $1,92 \%$ & $54,65 \%$ & $6,30 \%$ \\
\hline $\mathbf{7}$ & $8,62 \%$ & $21,39 \%$ & $1,32 \%$ & $1,86 \%$ & $48,84 \%$ & $11,70 \%$ \\
\hline $\mathbf{8}$ & $10,41 \%$ & $18,84 \%$ & $1,85 \%$ & $2,04 \%$ & $47,82 \%$ & $10,10 \%$ \\
\hline $\mathbf{9}$ & $16,08 \%$ & $15,78 \%$ & $0,77 \%$ & $0,67 \%$ & $53,81 \%$ & $7,07 \%$ \\
\hline $\mathbf{1 0}$ & $5,73 \%$ & $17,38 \%$ & $0,80 \%$ & $0,36 \%$ & $63,58 \%$ & $6,26 \%$ \\
\hline $\mathbf{1 1}$ & $9,48 \%$ & $15,39 \%$ & $1,73 \%$ & $0,20 \%$ & $63,29 \%$ & $7,42 \%$ \\
\hline
\end{tabular}




\begin{tabular}{|c|c|c|c|c|c|c|c|}
\hline 12 & $9,69 \%$ & $18,49 \%$ & $1,71 \%$ & $1,86 \%$ & $63,59 \%$ & $2,39 \%$ & $2,28 \%$ \\
\hline 13 & $15,61 \%$ & $20,96 \%$ & $3,00 \%$ & $0,26 \%$ & $50,16 \%$ & $2,55 \%$ & $7,46 \%$ \\
\hline 14 & $9,38 \%$ & $12,89 \%$ & $1,45 \%$ & $2,44 \%$ & $66,58 \%$ & $2,33 \%$ & $4,94 \%$ \\
\hline 15 & $31,75 \%$ & $15,87 \%$ & $0,68 \%$ & $8,14 \%$ & $39,87 \%$ & $0,68 \%$ & $3,02 \%$ \\
\hline 16 & $15,02 \%$ & $30,94 \%$ & $3,02 \%$ & $2,79 \%$ & $41,36 \%$ & $2,59 \%$ & $4,28 \%$ \\
\hline 17 & $11,13 \%$ & $17,77 \%$ & $1,65 \%$ & $11,53 \%$ & $50,14 \%$ & $1,61 \%$ & $6,17 \%$ \\
\hline Média & $12,88 \%$ & $17,94 \%$ & $1,94 \%$ & $2,49 \%$ & $55,06 \%$ & $4,90 \%$ & $4,78 \%$ \\
\hline
\end{tabular}

Observa-se que as rotas $2,4,9,10,12$ e 14 foram as rotas que apresentaram maior geração de resíduos orgânicos, com percentuais acima de 60\%, enquanto as rotas 1, 11, 15, 16 e 17 apresentaram os maiores percentuais de geração de resíduos potencialmente recicláveis (Papel/papelão, plástico, metal e vidro).

Nas localidades onde foram verificados os maiores percentuais de matéria orgânica são necessárias diversas ações de educação ambiental visando minimizar o desperdício de alimentos e o incentivo a compostagem doméstica. Adicionalmente, observou-se que parte destes resíduos é referente a podas e capinas do interior dos domicílios, sendo necessário por parte do poder público a criação de pontos de recebimento destes resíduos (também chamados de Ecopontos) os quais poderiam ser encaminhados para sistemas de compostagem em maior escala. Vale ressaltar que esta ação também deve ser acompanhada da conscientização da população, visando a obtenção de resultados satisfatórios.

As maiores quantidades de materiais recicláveis do tipo papel/papelão, plástico e metal, foram encontradas nas rotas 15 e 16, que correspondem aos bairros Setor Oeste, Jardim Neves, Jardim Marconal, Vila Morais, Jardim Morais, Vila Santa Barbara, Jardim Goiás e Setor Central I e II. Isso se deve ao fato de serem bairros centrais ou próximos ao centro, com uma quantidade razoável de comércios, que geram mais resíduos recicláveis do que bairros estritamente residenciais. Desta forma, os bairros que compõe estas rotas são propensos a ações mais intensas voltadas a redução da quantidade de materiais recicláveis nos resíduos domiciliares, como estratégias podem ser utilizadas pelo gestor municipal: i) instalação de pontos de entrega voluntário (PEVs), ii) intermediação e contato entre os comerciantes e lojistas com as cooperativas e associações de catadores do município, iii) desenvolver ações e campanhas de educação ambiental, iv) verificar a possibilidade de inserção de um regime tributário diferenciado para em empresas e comércios que aderirem a coleta seletiva.

\section{CONCLUSÕES}

A composição gravimétrica dos resíduos domiciliares do município de Rio Verde apresenta grande potencial de recuperação, vistos os elevados percentuais de matéria orgânica e recicláveis encontrados, que juntos totalizam 90 \% dos resíduos. Assim, ações voltadas ao tratamento desses resíduos utilizando técnicas como a compostagem e a reciclagem, por exemplo, seriam as soluções mais adequadas para a atual realidade do município.

Além da composição gravimétrica total dos resíduos no município, torna-se interessante a abordagem em cada setor (rota), visto as diferentes áreas e os perfis de renda da população, que influenciam diretamente sobre os hábitos de consumo e, consequentemente, sobre os resíduos gerados. Desta forma, 
alguns bairros se tornam mais propensos a abordagens voltadas a coleta seletiva e aproximação das associações e cooperativas de catadores, enquanto outras necessitam de maior conscientizam quanto a não geração e segregação dos resíduos.

Portanto, a composição gravimetria é de grande relevância no conhecimento do perfil dos resíduos produzidos em município, sendo possível a partir da mesma tanto a formulação de diretrizes gerais para como a adoção de medidas de caráter local, considerando as particularidades das diferentes regiões e populações que compõe a área urbana.

\section{REFERÊNCIAS}

ASTM. American Society for Testing and Materials. ASTM D 5231-92. International. Standard Test Method for Determination of the Composition of Unprocessed Municipal Solid Waste. ASTM, 2008.

ABNT. Associação Brasileira de Normas Técnicas. NBR 10007: Amostragem de resíduos sólidos. Rio de Janeiro: ABNT, 2004.

BRASIL. Lei n. 12.305, de 2 de agosto de 2010. Política Nacional de Resíduos Sólidos. Brasília: DOU, 2010.

DOMINGOS, D. C.; BOEIRA, S. L.. Gerenciamento de resíduos sólidos urbanos domiciliares: análise do atual cenário no município de Florianópolis. Revista de Gestão Ambiental e Sustentabilidade, v.4, n.3, p.14-30, 2015.

FRÉSCA, F. R. C.. Estudo da geração de resíduos sólidos domiciliares no município de São Carlos/SP, a partir da caracterização física. Tese (Doutorado) - Universidade de São Paulo, São Carlos, 2007.

FRÉSCA, F. R. C.; PUGLIESI, E.; MASSUKADO, L. M.; SCHALCH, V.. Determinação da composição gravimétrica dos resíduos sólidos domiciliares do município de São Carlos/SP. Revista DAE, São Paulo, n.178, p.48-57, 2008.

MACHADO, R. E.; RAFAEL, D. H.; CABRAL, S. M.; FIGUEIRÓ, P. S.. O empreendedorismo social como oportunidade de inclusão social: o caso de uma cooperativa de reciclagem. Revista de Gestão Ambiental e Sustentabilidade, v.8, n.1, n.1-18, 2019.

PASCHOALIN FILHO, J. A.; SILVEIRA, F. F.; LUZ, E. G.; OLIVEIRA, R. B.. Comparação entre as amassa de resíduos sólidos urbanos coletadas na cidade de São Paulo por meio de coleta seletiva de domiciliar. Revista de Gestão Ambiental e Sustentabilidade, v.3, n.3, p.19-33, 2014.

PESSOA, D. S.; COSME, A. M. F.; SILVA, V. F.; COSTA, R. O.; LIMA, V. L. A.; FERREIRA, A. C.. Resíduos Sólidos: evidências científicas sobre sua disposição final e impactos ambientais. Revista Ibero-Americana de Ciências Ambientais, v.9 n.7, p.202-210, 2018.

REZENDE, J. H.; CARBONI, M.; MURGEL, M. A. T.; CAPPS, A. L. D. A. P.; TEIXEIRA, H. L.; SIMÕES, G. T. C.; RUSSI, R. R.; LOURENÇO, B. L. R.; OLIVEIRA, C. A.. Composição gravimétrica e peso específico dos resíduos sólidos urbanos em Jaú (SP). Engenharia Sanitária e Ambiental, v.18, n.1, p.1-8, 2013.

SONTAG, A. G.; CRUZ, I. K. H.; CRUZ, F. P. B.; BERTOLINI, G. R. F.. Análise da viabilidade econômica para sistemas de tratamento de resíduos sólidos urbanos no município de Marechal Cândido Rondon/PR. Revista de Gestão Ambiental e Sustentabilidade, v.4, n.3, p.1-13, 2015.

A CBPC - Companhia Brasileira de Produção Científica (CNPJ: 11.221.422/0001-03) detém os direitos materiais desta publicação. Os direitos referem-se à publicação do trabalho em qualquer parte do mundo, incluindo os direitos às renovações, expansões e disseminações da contribuição, bem como outros direitos subsidiários. Todos os trabalhos publicados eletronicamente poderão posteriormente ser publicados em coletâneas impressas sob coordenação da Sustenere Publishing, da Companhia Brasileira de Produção Científica e seus parceiros autorizados. Os (as) autores (as) preservam os direitos autorais, mas não têm permissão para a publicação da contribuição em outro meio, impresso ou digital, em português ou em tradução. 